\title{
Designing Trousers to Limit the Burden on Upper Limbs of Non-professional Care Givers while Providing Nursing Care
}

\author{
Kazuaki URYU*, Megumi KOKATSU*, Satoshi HOSOYA* and Chiyomi MIZUTANI** \\ * Shinshu University, 3-15-1 Tokida, Ueda-shi, Nagano 386-8567, Japan \\ ** Otsuma Women's University, 12 Sanban-cho, Chiyoda-ku, Tokyo 102-8357, Japan
}

\begin{abstract}
In this research, movements of the upper limbs of caregivers when putting on / removing the dummy's nursing care clothes with different designs were measured using electromyography (EMG) and video filming. Caregivers' sensory assessments of the burden caused by their services were also investigated to determine how the physiological burden in the upper limbs and sensation of burden relate to each other. The subjects were 10 healthy women. None of the women had ever provided nursing care to anyone. For this experiment, four kinds of samples were used. By combining EMG and questionnaires, it was possible to quantify the burden on the upper limbs, which cannot be assessed solely according to work time. Differences in the workload associated with trousers having different designs were also studied. It is possible that by taking such an approach, nursing care clothes may be developed in such a way to further reduce the burden on caregivers while providing nursing care.
\end{abstract}

Keywords: Nursing Care, Trousers, Workload, Burden, EMG

\section{INTRODUCTION}

As the percentage of elderly persons in the population rapidly increases, nursing care has become a significant social issue in Japan. When preparing for the national examinations to obtain a license, nursing care workers need to acquire basic job techniques, ergonomic knowledge, and body mechanics that will help them to ease the burden of nursing care [1]. However, it is too much to ask the general public, who often provide home-based care for their family members, to acquire such basic techniques and expert knowledge [2]. As such, there has been growing demand for the development of nursing care goods that ordinary people can use effectively in their homes without a great deal of expert techniques or knowledge.

According to the guidelines set by the Ministry of Health, Labour and Welfare of Japan, daily nursing services for Level 3 - Level 5 recipients of nursing care ("bedridden persons") include meals [3], excretion, and changing clothes. Of these services, the caregiver must change the position of the recipient for excretion and changing clothes, and it is feared to cause strain in their lower back. Since such actions also involve procedures that use one's hands, a burden is placed on the upper limbs and shoulders of the caregiver as well.

Now, in many of the studies on nursing care movements and their associated burden, the strain on the lower back and the degree of burden on the entire body are assessed [4,5], whereas caregivers cannot carry out their jobs without using their upper limbs [6]. However, only a few focus on the measurement and assessment of the burden associated with such movements, with the upper limbs taken into consideration.

In this research, the workload of caregivers was assessed using electromyography (EMG) and video filming to measure the movements of their upper limbs when putting on / removing a dummy's nursing care clothing with different designs. The sensory assessments of caregivers' services were also investigated. If the relationship between movements of the upper limbs and the resultant burden can be made clear, it may be possible to gain knowledge that would be useful for designing nursing care clothes and diapers that can be easily put on and removed. Based on the assessment results, this research intends to gain knowledge that will be useful for designing nursing care trousers that help to reduce the burden on caregivers.

\section{METHODOLOGY}

\subsection{Subjects and experiment samples}

Subjects were 10 healthy women aged $23 \pm 3$ years, with a height of $159.7 \pm 8.7 \mathrm{~cm}$ and weight of $50.5 \pm 8 \mathrm{~kg}$. None of the women had ever provided nursing care to anyone. For this experiment, four kinds of samples were used: Sample 1 consisted of ordinary pajama-type trousers 


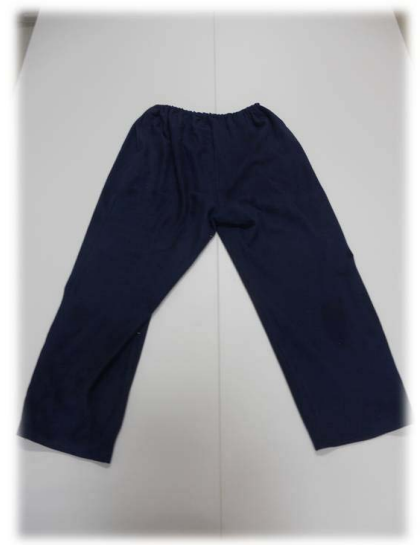

Figure 1: Sample 1

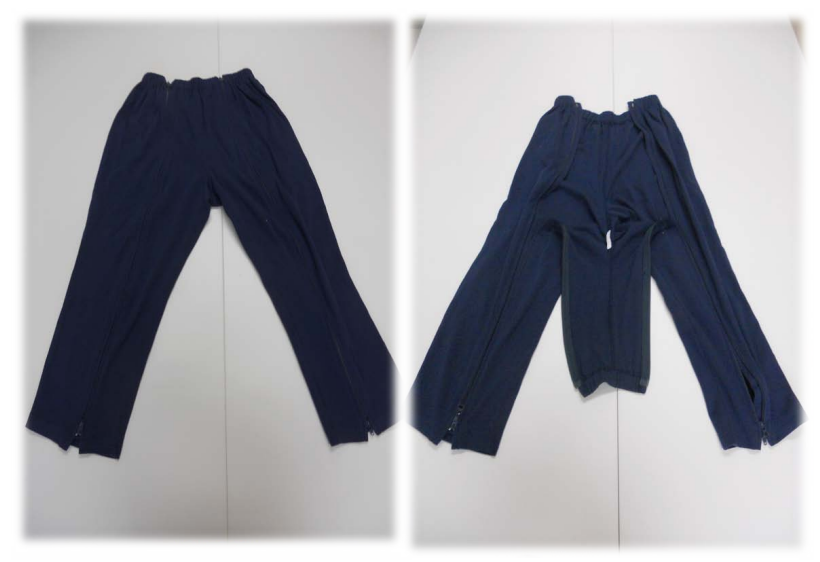

Figure 2: Sample 2

(Figure 1), Sample 2 was a pair of trousers with a fastener on the side of each leg (Figure 2), Sample 3 had an opening under the crotch secured by Velcro for ease of changing diapers, strings on both sides that could be tied at the center, and narrow cuffs (Figure 3), and Sample 4 was identical to Sample 3 except that its cuffs were wider (Figure 4).

\subsection{Experiment procedures and measurement parameters}

For this experiment, a silicon dummy named "Koharusan" (Mitaka Supply Co., Ltd.; Height: 150 cm; Weight: $12 \mathrm{~kg}$ ) was placed supine on a bed and the subjects were asked to put on and remove four different kinds of trousers on the dummy. Prior to the experiment, the subjects were briefed on the structures of each sample and the experiment procedures, and were given a chance to rehearse one time. To put on the clothes, the subjects were asked to put the sample that was laid out by the dummy's feet onto the dummy. To remove the clothes, the subjects were asked to take the sample that the dummy was wearing and place them by the dummy's feet. Each
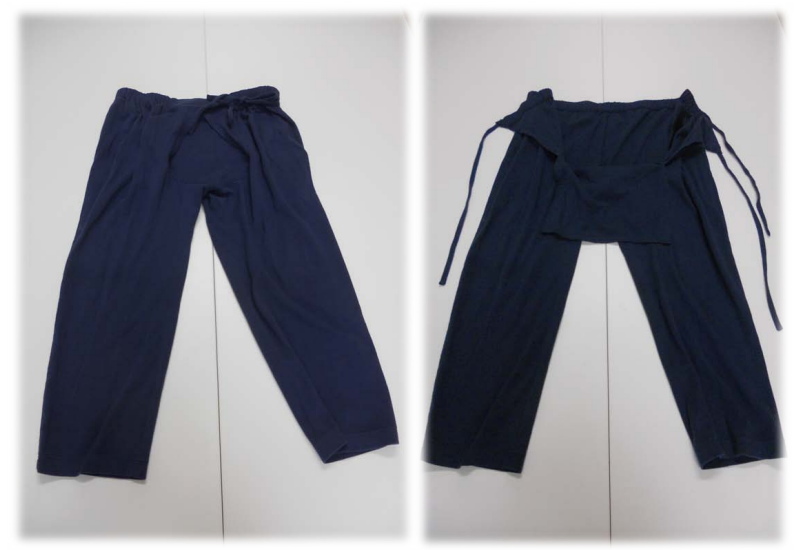

Figure 3: Sample 3
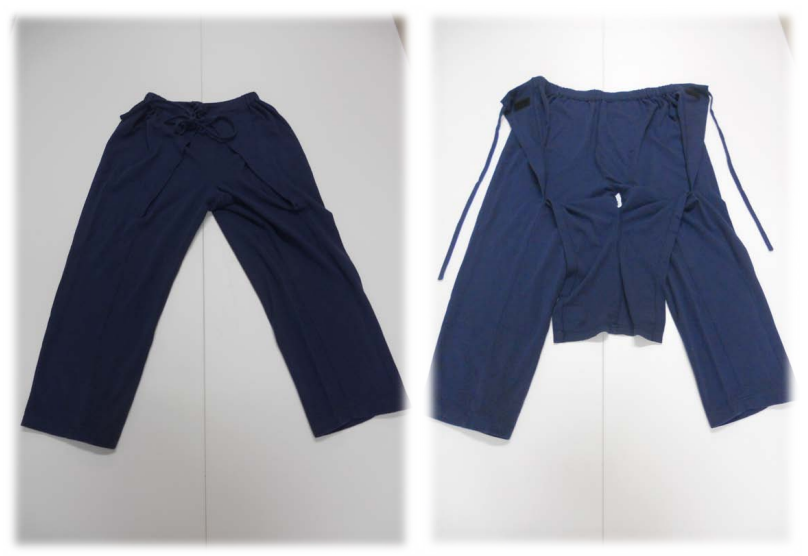

Figure 4: Sample 4

subject was asked to follow this process of putting on / removing the clothes once for each of the four samples (a total of eight procedures). The height of the bed was approximately $60 \mathrm{~cm}$ (Figure 5).

In order to assess the workload caused by movements during the procedures based on the amount of muscle activity, EMG measurements were taken from the right forearm at: 1 . the extensor digitorum muscle (function: dorsal flexion of the hand joint) and 2. the palmaris longus

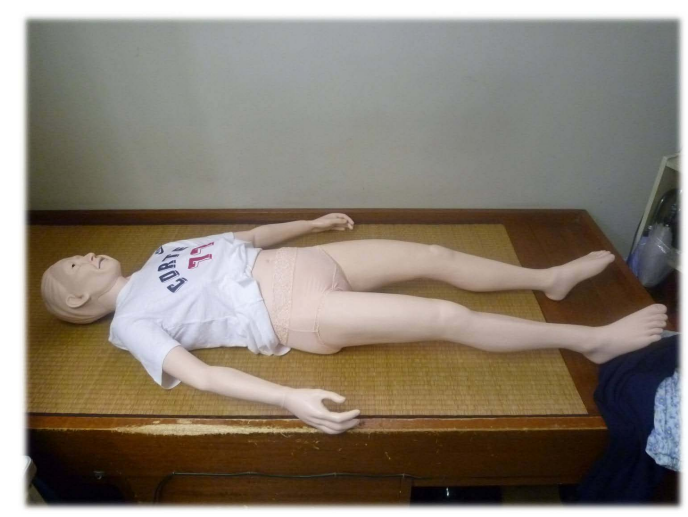

Figure 5: "Koharu-san" on the bed 


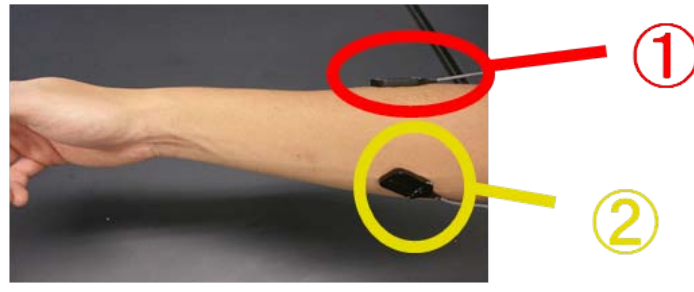

Figure 6: Electrode attachment points

muscle (function: palmar flexion of the hand muscle) (Figure 6). To analyze the procedures in detail, the process of the experiment was recorded on video (Panasonic DMC-FX-60) from the head of the dummy. After the experiment was over, in order to assess the sensation of burden from the set of movements, a questionnaire using the semantic differential (SD) method (scores: -2 to +2 pts.) was conducted for the four parameters of: ease of pulling up / down the trousers, fatigue when pulling up / down the trousers, ease of putting the trousers on around the hips, and fatigue when putting on the trousers around the hips. For EMG measurements, active electrodes (Delsys DE2-1) were used. Muscle potential signals were imported to a notebook PC at a sampling frequency of $1 \mathrm{kHz}$ via an A/D converter (BIOPAC System MP150).

\subsection{Analytical method}

For the purposes of this research, in order to assess the physiological burden of the subjects, time integrated values of rectified EMG (electromyogram) (Figure 7) and IEMG (integrated electromyogram) of the muscles being experimented on during the procedures were calculated, and were then standardized with the value of maximum voluntary contraction (MVC) (Figure 8) of voluntary movement as a reference to calculate a percentage of

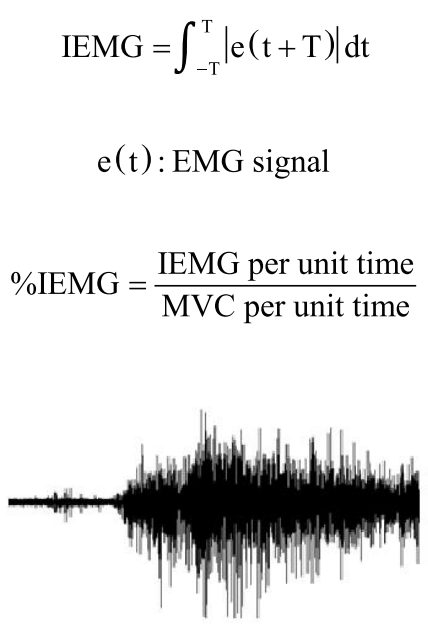

Figure 7: EMG data

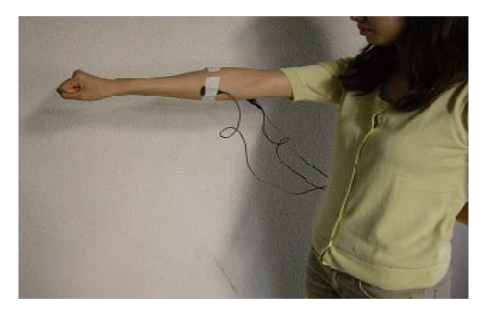

Figure 8: Voluntary movement for EMG standardization

IEMG (\%IEMG) per unit time. Additionally, in the three work processes described below, \%IEMG was calculated to assess workload. For voluntary movement, the subjects were asked to clench their fists with maximum force while reaching straight outward.

For video analysis, the time for putting on and removing nursing care trousers was measured using the recorded footage. The work process was divided into three parts and the times for each sub-process were compared: procedures around the ankles (Process 1), intermediate procedures from the ankles to the hips (Process 2), and finishing procedures such as tying the strings (Process 3). For Sample 2, Process 3 was finished when the work was completed with the fasteners closed, and for Samples 3 and 4, Process 3 corresponded to the procedures from tying the strings, etc. to completion of the job. When removing the trousers, the order of processes was reversed (Process 3 -> Process 2 -> Process 1), except that Process 3 is not necessary for removing Sample 1 (Process 2 -> Process 1).

For assessment of the sensation of burden, statistical analysis was made of scores from the four questions on the SD method questionnaire.

\section{RESULTS}

\subsection{EMG measurement}

Figure 9 shows the results of EMG measurement. The \%IEMG of the amount of muscle activity is shown on the vertical axis. The higher the value is, the greater the muscle load. If we look at each sub-process during the act of putting on the trousers, the values for both the extensor digitorum and palmaris longus muscles were highest for Sample 1 in Processes 1 and 2. The value of $\%$ IEMG for the extensor digitorum muscle was significantly higher than those for other samples. In Process 3, the \%IEMG values for both the extensor digitorum and palmaris longus muscles were significantly smaller for Sample 3. When removing the trousers, the value of $\%$ IEMG for the extensor digitorum muscle was significantly lower for Sample 3 in Processes 2 and 3, whereas 

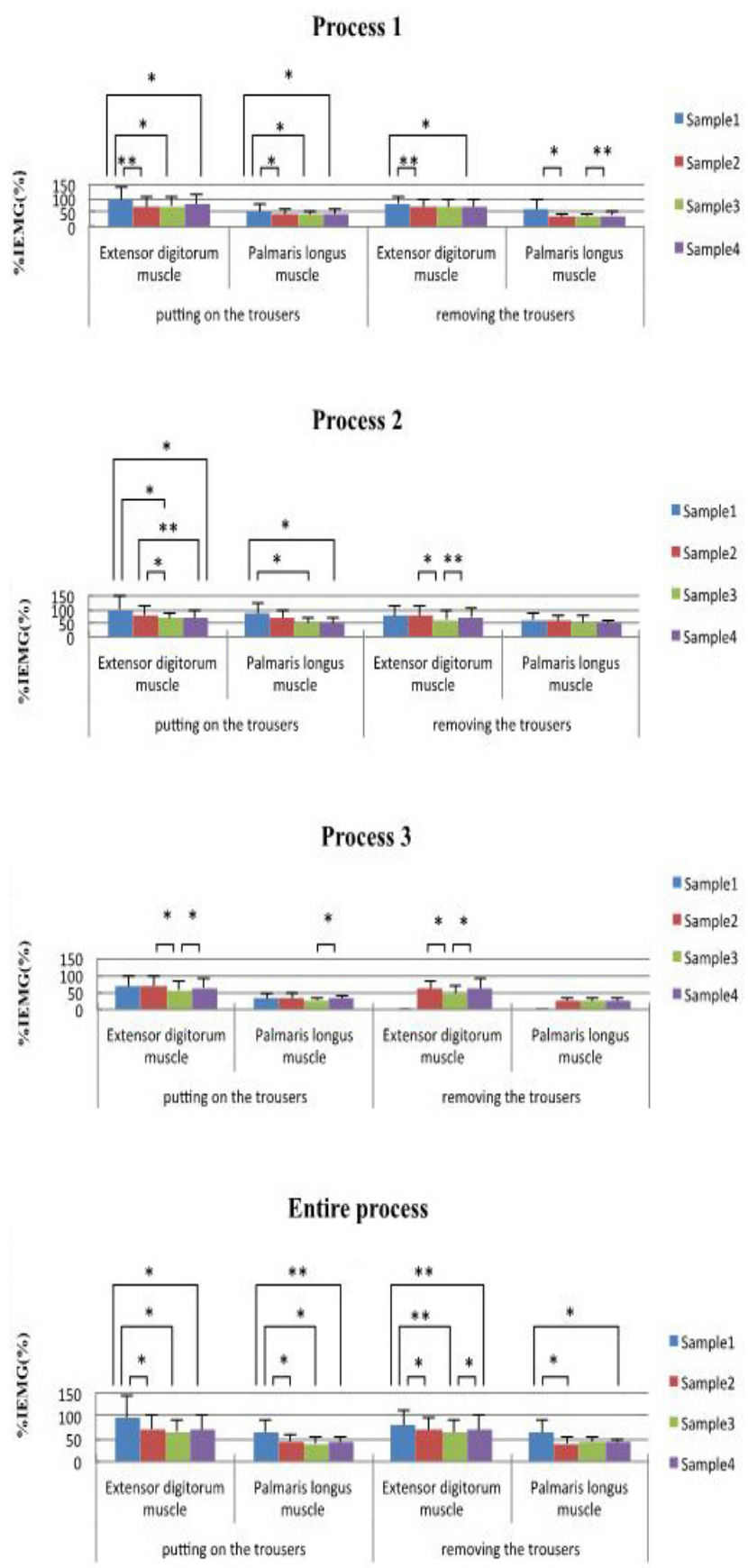

Figure 9: Comparison of \%IEMG

in Process 1 the values of \%IEMG for both the extensor digitorum and palmaris longus muscles were highest for Sample 1 and that for the palmaris longus muscle was lower than those of other samples for Sample 2. For the entire process, the values of \%IEMG for both the extensor digitorum and palmaris longus muscles were significantly higher than those of other samples for Sample 1 when putting on and removing the trousers. For the process of removal, the value of \%IEMG of the extensor digitorum muscle was significantly lower than that of other samples.

\subsection{Work time}

Analysis results of work time when putting on the trousers are shown in Figure 10, and those for removing the trousers are shown in Figure 11. When putting on the trousers, the work times for Sample 2 during Processes 1 and 2 were significantly shorter than those for other samples, while the time was significantly longer during Process 3. For the entire process, no significant differences could be observed among the different samples. When removing the trousers, the work time for the entire process for Sample 1 was significantly shorter than those for other samples, since it did not include Process 3. The work time for the entire process for Sample 4 tended to be long because Process 2 was time-consuming.

\subsection{Sensation of burden}

Shown in Figures 12-14 are the average scores of each sample in the questionnaire. For all of the questions, Sample 1 had the lowest score. For the five questions that did not ask about fatigue felt when pulling down the trousers, there were significant differences in scores between Sample 1 and other samples. This shows that Sample 1 caused greater fatigue than the others, as it was harder to put on and remove.

Sample 2 was given a lower score than Samples 3 and 4 for ease of putting on the trousers around the hips (Figure 14). For fatigue felt when pulling up the trousers, too, Sample 2 received a lower score than Sample 3 (Figure 12). These results show that procedures around the hips were more difficult for Sample 2 than for Samples 3 and 4.

Sample 4 had a larger opening under the crotch than Sample 3, but there were no significant differences between the two in terms of scores for fatigue felt during this experiment.

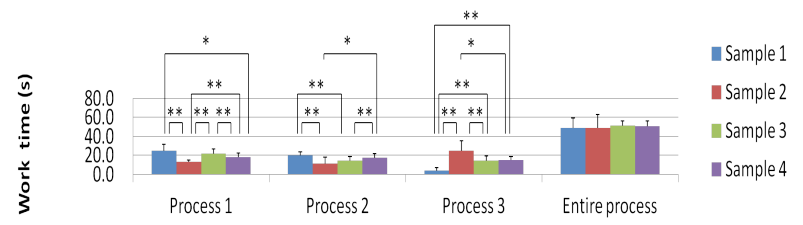

Figure 10: Average work time for each sample (when putting on the trousers)

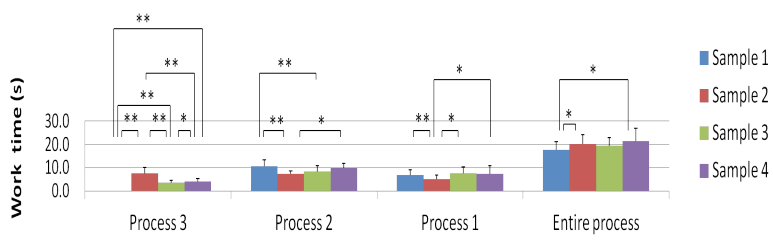

Figure 11: Average work time for each sample (when removing the trousers) 

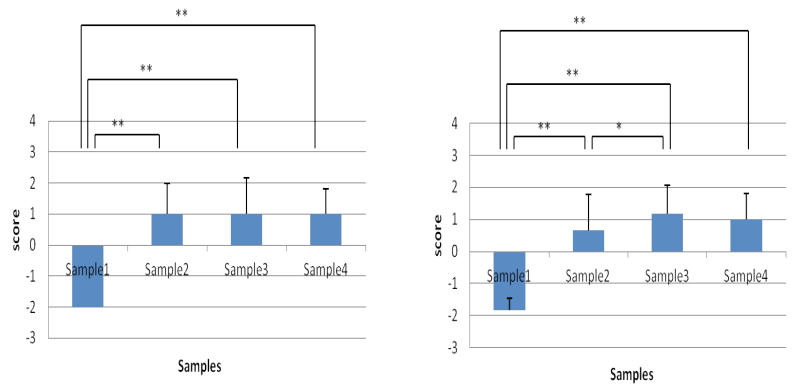

Figure 12: Questionnaire responses on pulling up the trousers (left: ease, right: fatigue)

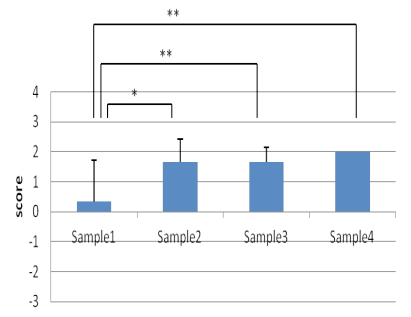

Samples

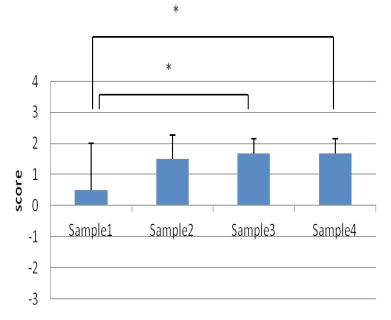

Samples
Figure 13: Questionnaire responses on pulling down the trousers (left: ease, right: fatigue)
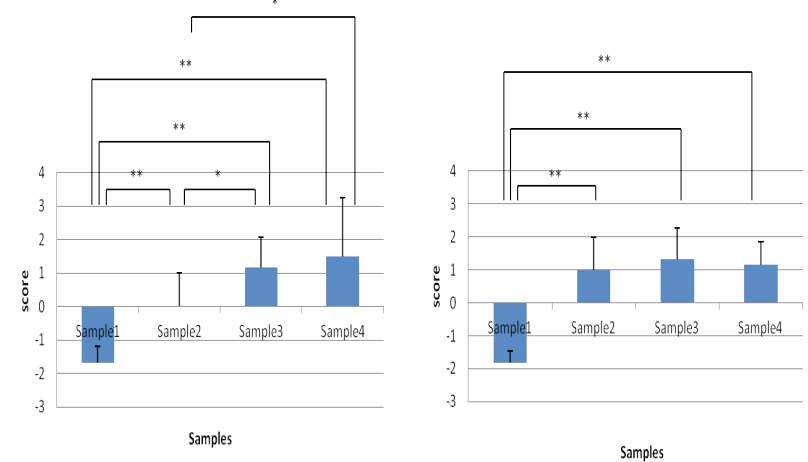

Figure 14: Questionnaire responses on work around the hips (left: ease, right: fatigue)

\section{CONSIDERATIONS}

\subsection{Assessment of the burden on the upper limbs when putting on / removing the clothes}

As shown in Figures 10 and 11, comparisons of the work times for four different samples throughout the entire process did not show any significant differences, and so no one burden appears heavier than any other. If attention is paid to the activities of the two muscles in the upper limbs when putting on / removing the clothes, however, the amount of such activities for Sample 1 demonstrated significant differences compared to the other samples. Also, the \%IEMG value for Sample 1 was high, which suggests that the physiological burden on the upper limbs was great.
If muscle activity (\%IEMG) is compared when putting on / removing the clothes in each sub-process of putting on the clothes, Sample 1 showed significant differences from other samples for both muscle activities for Process 1, Process 2, and the entire process, and so it is safe to say that Sample 1 results in heavy muscle load. In a similar vein, a comparison among the samples for all of the sub-processes showed that Sample 1 put greater load on the muscle for the entire process. As described thus far, physiological and by-process analyses have made it possible to make a quantitative comparison of the burden on the upper limbs, which could not be assessed based solely on the work time. Also, the results of subjective assessment revealed that Samples $2-4$ earned higher scores than Sample 1 for most of the parameters, which leads to the belief that the physiological burden and psychological assessment largely correspond to each other. In light of this, it would be important to combine physiological measurement (EMG) and psychological assessment (questionnaires) when making qualitative evaluations of the burden caused by movements when providing nursing care.

\subsection{Designing nursing care trousers}

As the Sample 1 results shown in Figure 9 indicate, a large burden was placed on the upper limbs during Processes 1 and 2 when putting on the nursing care trousers. In other words, it is assumed that the procedure of pulling up the trousers and pushing the hips into them has an influence on the physiological burden experienced. This also points to the importance of the trousers' design from the waist to thighs. It is therefore believed that, if an opening is made in the upper part of the trousers as in Samples 3 and 4, a moderate degree of freedom will be allowed when conducting the procedure around the hips, thereby reducing the workload.

Concerning the burden on the upper limbs, there is a possibility that movements like lifting the body, rather than tying the drawstrings or other precision work, place a burden on caregivers both physiologically and psychologically. As such, it is assumed that designing trousers that decrease movements such as lifting the body to change the nursing care recipients' positions would be effective in reducing the burden on the upper limbs when putting on / removing nursing care trousers.

Based on the above analysis, two types of nursing care trousers that help to reduce the burden are offered below. 


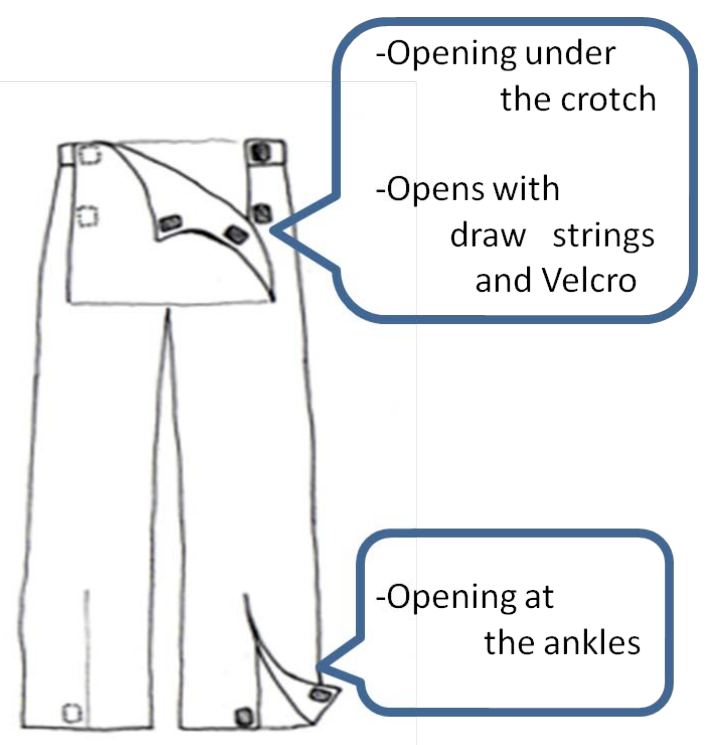

Figure 15: Design of nursing care trousers for reducing muscle load

\subsubsection{Nursing care trousers design plan 1}

Shown in Figure 15 is a nursing care trousers design created based on Sample 3, which was shown to involve less of a burden during Processes 2 and 3. To reduce the muscle load during Process 1, it is designed with an opening at the ankles. Providing an appropriately sized opening at the ankles and hips is intended to reduce muscle burden during the act of putting them on.

\subsubsection{Nursing care trousers design plan 2}

Shown in Figure 16 is a nursing care trousers design created based on Sample 2, which took less work time during Processes 1 and 2. To shorten the work time during Process 3, drawstrings and Velcro are provided instead of fasteners for the openings at the front of both legs. Providing large openings that stretch from the hips to ankles at the front of both legs is intended to shorten the work time for putting on the trousers from the ankles to hips. Although the large openings on the front of both legs require additional time for finishing, this is compensated for through the use of drawstrings and Velcro, which can be easily closed.

\section{CONCLUSION}

In this research, the burden on the upper limbs when putting on / removing nursing care trousers was analyzed through EMG measurement, questionnaires, and video filming. By combining EMG and questionnaires, it became possible to quantify the burden on the upper limbs, which cannot be assessed solely according to

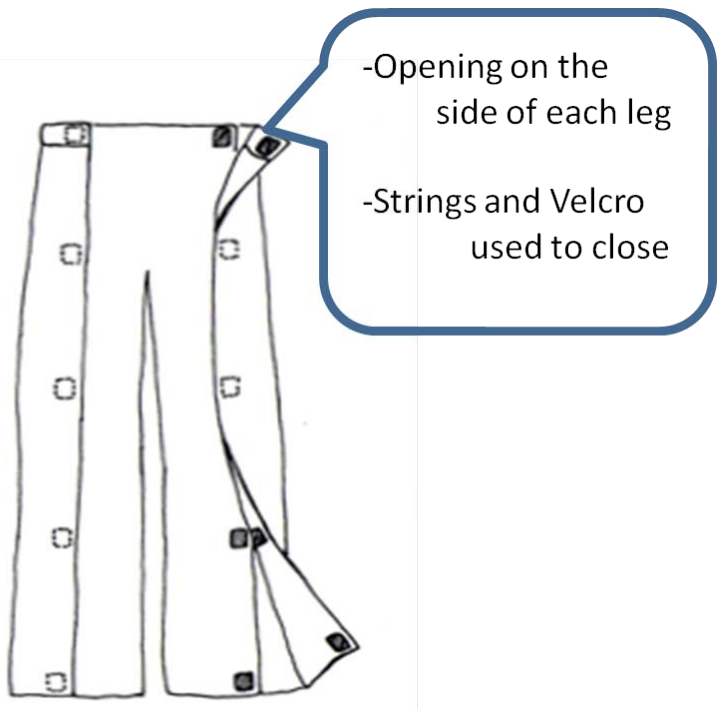

Figure 16: Design of nursing care trousers for work time reduction

work time. Based on these assessment results, the key design points for reducing workload were identified and two design proposals were made for nursing care trousers. It is possible to suggest that by taking this kind of approach, nursing care products may be developed that they can further reduce the burden on caregivers while providing nursing care. And beyond the field of nursing care, there are additional fields that this approach could be applied to, such as sportswear or workplace clothing where comfort can be increased and muscle burden can be decreased.

\section{ACKNOWLEDGEMENT}

This work was supported by JSPS KAKENHI (22300249).

\section{REFERENCES}

1. Information on

http://www.mhlw.go.jp/kouseiroudoushou/ shikaku_shiken/kaigohukushishi/index.html

2. Nagase M; Transfiguration of main caregiver's recognition and/or action in the home switchover, Bulletin of Health Sciences Tokai University, 8, pp.89-95, 2002.

3. Information on http://www.mhlw.go.jp/topics/kaigo/nintei/gaiyo2.html

4. Kamioka H, Okuizumi H, Okada S, Watanabe S, Takahashi R, Handa S, Ohshio T, Honda T, Moriyama S, Kitayuguchi J, Kamada M, Mutoh Y; A cross- 
sectional study of actual conditions and related factors of low back pain among female caregivers in special nursing homes for the elderly, J. Agric. Sci., Tokyo Univ. Agric., 55(1), pp.38-44, 2010.

5. Tomioka K; Low back pain among care workers working at newly-built nursing homes for the aged, Japan Society for Occupational health, 50(3), pp.8691, 2008.

6. Menzel, N.N.; Back pain prevalence in nursing personnel: measurement issues, AAOHN-J, 52, pp.5465, 2004.

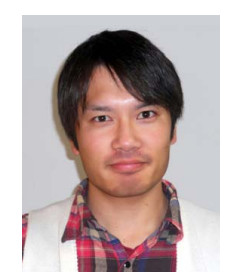

\section{Kazuaki URYU}

Kazuaki Uryu is a master course student in the Graduate School of Science and Technology at Shinshu University. His research field is Affective Engineering and motion analysis.

\section{Megumi KOKATSU}

Megumi Kokatsu is a master course student in the Graduate School of Science and Technology at Shinshu University. Her research field is Affective Engineering, specifically wearing comfort and the ease of movement of clothing.

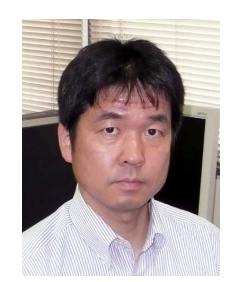

\section{Satoshi HOSOYA}

Satoshi Hosoya received the Doctor of Engineering degree in Affective Engineering from Shinshu University, Japan in 2002. He is an Associate Professor in the Faculty of Textile Science and Technology at Shinshu University. His research interests include evaluation methods of product usability and human centered design.

\section{Chiyomi MIZUTANI}

Chiyomi Mizutani received a Ph.D degree from Shinshu University and another PhD degree from Mukogawa Women's University. She is an Associate Profesor in the Textile Clothing Science Department at Otsuma Women's University. Her main research areas are the evaluation of deodorant materials and the application of functional materials for nursing care. 